\title{
Cost and fault-tolerant aware resource management for scientific workflows using hybrid instances on clouds
}

\author{
Vinay K. ${ }^{1}$ (D) . S. M. Dilip Kumar ${ }^{1}$. Raghavendra S. ${ }^{1}$. \\ Venugopal K. R. ${ }^{1}$
}

Received: 8 July 2017 / Revised: 12 September 2017 / Accepted: 12 October 2017/

Published online: 23 October 2017

(C) Springer Science+Business Media, LLC 2017

\begin{abstract}
Cloud service providers are offering computing resources at a reasonable price as a pay-per-use model. Further, cloud service providers have also introduced different pricing models like spot, blockspot and spotfleet instances that are cost effective and user's have to go through the bidding to balance the reliability and monetary costs. Henceforth, Scientific Workflows (SWf) that are used to model applications of high throughput, computation and complex large-scale data analysis are significantly adopting these computing resources. Nevertheless, spot instances are terminated when the market spot price exceeds the users bid price. Moreover, failures are inevitable in such a large distributed systems and often pose a challenge to design a fault-tolerant scheduling algorithm for SWf. This paper presents an efficient, low-cost and fault-tolerant scheduling algorithm and a bidding strategy to minimize the volatility and cost of resource provisioning for SWf. The proposed algorithm uses spot and blockspot instances as hybrid instances in comparison with on-demand instance to reduce the execution cost and fault-tolerant while meeting the SWf deadline. The results obtained reveal the promising potential of the proposed scheduling algorithm and are demonstrated through empirical simulation study that is robust under short deadlines with minimal makespan and cost.
\end{abstract}

\section{Vinay K.}

ec.vinay@gmail.com

S. M. Dilip Kumar

dilipkumarsm@gmail.com

Raghavendra S.

raghush86@gmail.com

Venugopal K. R.

venugopalkr@gmail.com

1 University Visvesvaraya College of Engineering, K R Circle, Dr Ambedkar Veedhi, Bengaluru, KA 560001, India 
Keywords Cloud computing $\cdot$ Scientific workflows $\cdot$ Scheduling $\cdot$ Spot $\cdot$ Blockspot On-demand · Instances · Fault-tolerant

\section{Introduction}

Scientific workflows are composed of many fine computational granularity tasks that are control and data dependent [13]. These SWf are used to execute a high throughput computing and data analysis. Diverse disciplines use SWf to conduct large scale scientific computations. SWf enable scientists to comfortably define computational tasks and their dependencies in an easy way that increases the applications performance and reduces the time required to get the scientific results [14].

SWf are increasingly migrating towards cloud computing that provides computing resources (e.g., servers, storage and networks) at a lower cost on an as-needed basis. Although, the pricing of these cloud resources can vary dynamically depending on the supply-chain and demand [25]. Scheduling SWf on the cloud significantly reduces the computation cost and makespan, but is also prone to resource failures. Failures are usually due to hardware faults, software faults, failures due to virtualization, etc. The failures in the point of SWf are categorized as task failures, Virtual Machine (VM) failures and SWf level failures [10]. However, failures are uncertain while running SWf that consists of thousands of tasks.

The Amazon Web Services (AWS) ${ }^{1}$, which is a pioneer in providing on-demand cloud computing infrastructure began with the on-demand pricing model and later added reserved and spot pricing model as depicted in Fig. 1. The spot pricing model is further classified into Spotblock and Spotfleet pricing models for cost effective computation.

In December 2009, $\mathrm{AWS}^{2}$ released spot instances (SI), an unused Amazon EC2 capacity for customers whose price is $70 \%$ lower than the on-demand instances. The SI price dynamically changes based on the supply and demand, and are procured through bidding. Customers acquire SI through a bidding process and pay on an hourly basis. It runs until the current SI price exceeds the bid price and later terminates exactly after 2 minutes. Even though there is a significant reduction in the cost, but with low Quality of Service (QoS) and is also susceptible to out-of-bid events which made SI a less reliable pricing model. There is a trade-off between the QoS and cost of the instances [12]. An efficient, fault-tolerant and robust scheduling algorithm is essential to mitigate such resource failures. Moreover, SWf can be greatly benefited from using SIs (spot instances) with optimal bidding strategy and an effective fault-tolerant mechanism. In October 2015, Amazon Web Services launched blockspot $^{3}$ (i.e., SIs with a defined duration of workloads) where the customers can bid SIs for a duration of 1 hour to 6 hours whose price is slightly higher than SIs and 45\% lower than the on-demand instances and once procured these instances will be reserved for the next 1 hour to 6 hours based on the requirement without interruption.

This paper presents a scheduling heuristic, fault-tolerant mechanism and an optimal bidding strategy using hybrid instances such as, spot, blockspot and on-demand instances to schedule SWf efficiently. It minimizes the execution cost and also provides robust schedule to meet the deadline constraint of SWf.

\footnotetext{
${ }^{1}$ http://aws.amazon.com

${ }^{2}$ https://aws.amazon.com/ec2/spot/

${ }^{3}$ https://aws.amazon.com/ec2/spot/pricing/
} 


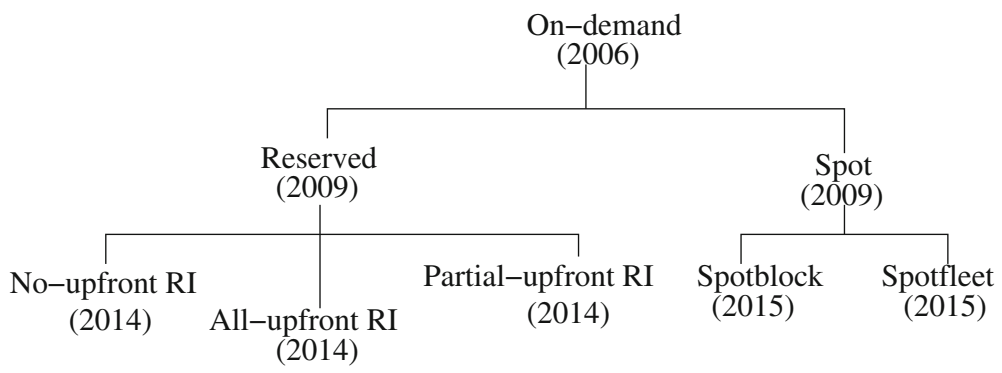

Fig. 1 Amazon Web Services Cloud Pricing Models

\subsection{Contributions}

- A scheduling heuristic that uses spot, blockspot and on-demand resources to schedule SWf in a cost efficient manner.

- Determine a runtime estimation of SWf dependent tasks on a particular instances using a stochastic approach.

- A fault-tolerant system model to mitigate resource failures/out-of-bid events during the scheduling of SWf.

- An optimal bidding strategy for spot and blockspot instances using spot price history in order to acquire resources.

The rest of this paper is organized as follows. Section 2 presents a review of related literature. Background of the proposed approach is discussed in Section 3. Proposed system models and approaches are explained in Sections 4 and 5. While the performance evaluation and discussions are reported in Sections 6 and 7 contain the conclusions.

\section{Related works}

Branches of cloud computing literature review include cloud computing economics, resource management, bidding strategies and fault-tolerant mechanisms. Cloud resource failures and its performance variations demand for fault-tolerance while scheduling SWf. In [8] it has been discussed that Virtual Machines (VMs) performance varies for different instance types, availability zones, data centers and even different times of the day.The work in [15] emphasize on the need for fault-tolerance in running SWf in cloud environments. The work in [20] proposes a Petri net model for small business applications using spot instances in public clouds to reduce the cost. It is based on the decision making framework, takes as input a list of cost elements, a list of qualitative criteria, weights and generates as output scores for the cloud. The authors in [19] utilized spot instances to provision web applications and the proposed approach reduced the resource cost significantly with minimal Quality-of-Service (QoS) in terms of availability and response time and the work is restricted to only fault-tolerant applications. The authors in [9] proposed LLOOVIA, an optimization technique which was designed for optimal allocation of the VMs required for the different workloads. The reserved and on-demand instances are considered in this work that are much costlier as compared against spot and blockspot instances. The work in [26] designed a fault-tolerant model that extends the traditional Primary Backup model in which two copies of one task are allocated to two different VMs. Even though the proposed 
approach achieves high QoS, but the additional resource cost of the overall execution cost is the downside of this work. The work in [16] proposed a SWf-level data placement model to reduce the data transfer cost. In this approach multiple SWf are considered as a whole and dynamically distributed to appropriate data centers, which consumes additional resource cost but achieves high QoS. The work in [16] proposed a dependable scheduling strategy based the on game-theory to enhance system availability and the work has achieved reasonable data replication. Enhanced adaptive incremental checkpointing for multi-threaded applications under spot instance pricing is proposed in [11] to lower the monetary costs.

Well known fault-tolerant mechanisms that can handle failures are alternate resource, checkpointing, retry and replication [5]. Replication is the fundamental in facilitating fault tolerance in space and time shared fashion [17]. Further, estimating runtime of the task is a paramount in a distributed system that minimizes the schedule length to meet hard deadlines [7, 23]. The tasks are replicated in idle time slots to reduce the cost and schedule length [4]. Tasks are replicated in either idle time slots or solely on the new resources; some schedules use a hybrid approach in replicating of tasks on both idle time slots and new resources to achieve high fault tolerance.

\section{Background preliminaries}

In this section, we describe some of the important terminologies, metrics and concepts used in the successive sections.

A SWf (Scientific Workflows) consists of a set of precedence constrained tasks represented by a Directed Acyclic Graph (DAG), $G_{w}=\left(T_{w}, E_{w}\right)$, where $T_{w}=\left\{t_{1}, t_{2}, . ., t_{p}\right\}$ is a set of precedence constrained tasks. There exists edges $E_{w}$ such that $e_{i, j}=\left(t_{i}, t_{j}\right)$ is a dependency edge between tasks $t_{i}$ and $t_{j}$, in which $t_{j}$ is said to be parent of $t_{i}$ and $t_{i}$ is the child of $t_{j}$. Each task $t_{i}$ is executed by determining its parent tasks, more accurately the one that completes the communication at the latest time [24]. The Earliest Start Time (EST) and Earliest Finish Time (EFT) of the task $t_{i}$ respectively are defined in (1) and (2):

$$
\begin{aligned}
\operatorname{EST}\left(t_{i}\right)= & \begin{cases}0, & \text { if } t_{i}=t_{0} \\
\max _{t_{p} \in P_{i}} \operatorname{EST}\left(t_{p}\right)+e_{p}, & \text { otherwise }\end{cases} \\
& \operatorname{EFT}\left(t_{i}\right)=\operatorname{EST}\left(t_{i}\right)+e_{i}
\end{aligned}
$$

where $t_{0}$ is an entry task without any predecessors, $t_{p}$ is the parent task of $t_{i}, P_{i}$ is the set of parent tasks of $t_{i}$ and $e_{p}$ and $e_{i}$ are the execution time of $t_{p}$ and $t_{i}$ respectively. Each SWf is specified by a user specified deadline $W_{D}$ and each SWf tasks $T_{w}$ has a respective task lengths $l e n_{i}$ used to estimate the tasks execution time before scheduling.

Critical path $C_{p}$ is the overall path of the SWf from start node to the exit node and also it determines the makespan of the SWf.

Some of the definitions related to $C_{p}$ are given below

- Minimum Execution Time (MET):

$$
\operatorname{MET}\left(t_{i}\right)=\min _{s \in S_{i}} E T\left(t_{i}, s\right)
$$

Equation (3) defines the MET of task $t_{i}$, where ' $s$ ' is the instance type such as small, medium, large, xlarge and so on, $S_{i}$ is the instance pricing model, such as on-demand, spot and blockspot and ET is the execution time of task $t_{i}$ on 's' 
- $\quad$ Minimum Transfer Time (MTT):

$$
\operatorname{MTT}\left(e_{i, j}\right)=\min _{r \in S_{j}, s \in S_{i}} T T\left(e_{i, j}, r, s\right)
$$

Equation (4) defines the Minimum Transfer Time required to transfer data from one instance $S_{j}$ which is executing task $t_{j}$ to another instance $S_{i}$ which is executing task $t_{i}$, where $r$ and $s$ are the two different instance types and $T T$ is the data transfer time between the task $t_{j}$ and $t_{i}$.

- $\quad$ Earliest Start Time (EST):

$$
\begin{gathered}
E S T\left(t_{\text {entry }}\right)=0 \\
\operatorname{EST}\left(t_{i}\right)=\text { max }_{t_{p} \in \operatorname{predessor}\left(t_{i}\right)} \operatorname{EST}\left(t_{p}\right)+\operatorname{MET}\left(t_{p}\right)+\operatorname{MTT}\left(q_{p, i}\right)
\end{gathered}
$$

EST is an earliest start time of a task and is given by the (6), where $t_{p}$ is the parents of task $t_{i}$. The Earliest Finish Time (EFT) which is the time a task can finish its execution is defined by the (7).

$$
\operatorname{EFT}\left(t_{i}\right)=\operatorname{EST}\left(t_{i}\right)+\operatorname{MET}\left(t_{p}\right)
$$

Latest Finish Time (LFT) is the time a task has to finish its execution within the deadline $W_{D}$. It is defined by the (8) and (9).

$$
\begin{gathered}
\operatorname{LFT}\left(t_{\text {exit }}\right)=W_{D}, \\
\operatorname{LFT}\left(t_{i}\right)=\min _{t_{s} \in \operatorname{successor}\left(t_{i}\right)} \operatorname{LFT}\left(t_{s}\right)-\operatorname{MET}\left(t_{s}\right)-\left(q_{p, i}\right)
\end{gathered}
$$

where successor $t_{i}$ is all the children nodes of $t_{s}$. Henceforth, the tasks that do not have slack time to finish their execution, those particular tasks are considered to be critical tasks [1] and is visualised in Fig. 5a. The proposed algorithm schedule these critical tasks onto an instance that offer a low execution time.

Latest Time to BlockSpot (LTBS) is the latest time an algorithm shifts the SWf task to blockspot instances to meet the deadline constraint. The proposed algorithm make the most of spot market before the time flag, LTBS and shifts to blockspot instance later. LTBS facilitate in choosing the appropriate instance to speed up the SWf execution. For every ready task, the scheduling decisions are made based on current time $t$ and the LTBS. LTBS at time $t$ is the difference between the deadline $W_{D}$ and the critical path at time $t$ i.e., LT B $S_{t}=W_{D}-C_{p_{t}}$.

Resource pricing models In the proposed model, three types of Amazon EC2 instances are used which vary in their pricing as follows:

(i) Spot Instance: Users have to bid for the spare Amazon EC2 instances and it is only available when user's bid price exceeds the current spot market price and lasts as long as their bid price is higher than the current spot price or user choose to terminate it. A new feature of 2 minutes time period is provided in SI to save the status of ongoing tasks on a running instances before termination. The spot price fluctuates very often based on the supply and demand of Amazon EC2. The spot price is different for different instances and varies between regions and availability zones.

(ii) On-Demand Instance: Users are allowed to use the dedicated instances on an hourly basis with no long-term commitments, whose price is higher than the SI but reserves the instances for defined hours. The advantage of using on-demand instances is, the user need not participate in bidding for acquiring instances and also there is no prior instance termination. 


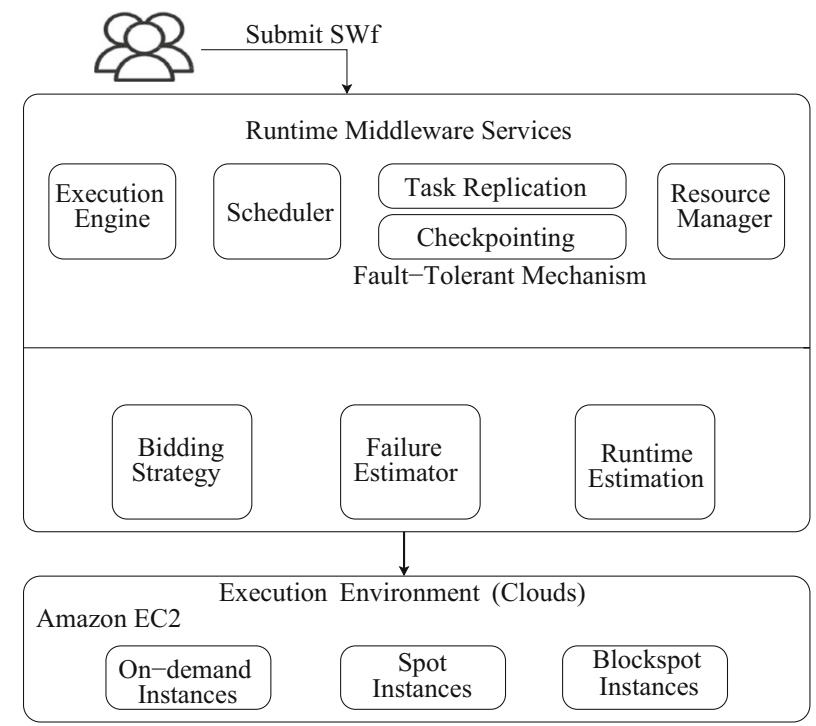

Fig. 2 Proposed Architecture of Fault-Tolerant SWf Management System

(iii) BlockSpot Instance: It is a newly introduced instance, which is a combination of spot and on-demand instances. The price is slightly higher than SI and lower than the ondemand instance. The user has to bid for the required instances on an hourly basis. User can bid the unused Amazon EC2 for 1 hour to 6 hours, the advantage of using blockspot instance is the price (lower than the on-demand) and there cannot be an out-of-bid for at least 6 hours. An efficient scheduling algorithm is required to plan and choose appropriate instances to minimize the execution cost.

Total Execution Cost (TEC) It is the total cost of all the instances utilized during the execution of SWf tasks. The cost of individual instance is calculated as per the Amazon's pricing model $^{4}$. For example, if the spot instance is used during the execution of SWf tasks, then that particular instance price is considered in the execution cost.

\section{System model}

The proposed system architecture of a fault-tolerant SWf management system is depicted in Fig. 2. It consists of two layers, namely, runtime middleware services and execution environment (clouds). The runtime middleware services act as an interface between the user application and the cloud. Users submit the SWf onto the runtime middleware services which schedules the SWf tasks, handles fault tolerant mechanism, and allocates resources in a cost efficient manner.

\footnotetext{
${ }^{4}$ http://aws.amazon.com/ec2/purchasing-options/spot-instances/
} 
The tasks and their dependencies in SWf are managed by the execution engine and also ensures that the task is only terminated when all of its parent tasks have completed its execution successfully. Execution engine mainly depends on the computing resources such as compute, network and storage as defined in the SWf execution and releases only free tasks to the scheduler. A delay can be introduced by configuring an execution engine by users to assure the SWf scheduling and execution are not overloaded.

The scheduler is a type of global task scheduler and deals with mapping and managing interdependent tasks of SWf on distributed resources. Furthermore, it introduces a queue delay which is the total amount of time taken between the task submitted to the scheduler and its execution in a worker node.

Fault-Tolerant Mechanism Resource failures are considered in this work and generally failures can be hardware, software or any other faults in the execution environment that leads to interference in the task execution. Resource failures also occur from out-of-bid events among SIs. The runtime middleware services dwell with these failures having task replication and checkpointing as fault-tolerant mechanisms. In distributed systems, failures is shown to follow a Weibull distribution [18]. In this work, the Weibull distribution is used to model resource failures that employ the task replication and checkpointing. Tasks are replicated on idle instances based on the deadline constraint and checkpointing is used to save the states of a running process periodically.

The Resource manager negotiates, reserve and allocate resources that are required for the execution of SWf on cloud and it also manages Virtual Machines (VMs) for different SWf.

$$
P(\text { out }- \text { of }- \text { bid } \mid \text { price })=\frac{P(\text { price } \mid \text { out }- \text { of }- \text { bid }) P(\text { out }- \text { of }- \text { bid })}{P(\text { price })}
$$

Failure Estimator estimates the Failure Probability (FP) of a particular in-bid price using Naive Bayes model [22]. To calculate the FP of an unknown instance, i.e. out - of - bid (the in-bid price that might go out-of-bid) and is classified as class out - of - bid with

Table 1 Nov 2016 Month Spot and Blockspot Instance Characteristics of Amazons US East (North Virginia) region

\begin{tabular}{lllll}
\hline $\begin{array}{l}\text { Instance } \\
\text { Types }\end{array}$ & $\begin{array}{l}\text { Average Spot } \\
\text { Price per } \\
\text { hour }\end{array}$ & $\begin{array}{l}\text { Average Blockspot } \\
\text { Price per } \\
\text { hour }\end{array}$ & $\begin{array}{l}\text { Frequency of Spot } \\
\text { instance being } \\
\text { out-of-bid (month) }\end{array}$ & $\begin{array}{l}\text { Cost-Savings } \\
\text { over } \\
\text { on-demand }\end{array}$ \\
\hline m1.small & 0.0071 & 0.024 & Low & $63 \%$ \\
m1.medium & 0.0085 & 0.048 & Low & $67 \%$ \\
m1.large & 0.0165 & 0.096 & High & $68 \%$ \\
m1.xlarge & 0.0323 & 0.193 & Low & $67 \%$ \\
m3.xlarge & 0.0412 & 0.146 & High & $81 \%$ \\
m3.2xlarge & 0.0978 & 0.293 & High & $79 \%$ \\
m2.xlarge & 0.0166 & 0.135 & High & $90 \%$ \\
m2.2xlarge & 0.0376 & 0.27 & Low & $91 \%$ \\
m2.4xlarge & 0.0842 & 0.539 & High & $88 \%$ \\
\hline
\end{tabular}


possible outcomes (Low/High) and the probability of task failure or success is based on the spot price history as discussed in Table 1. The one month's SI price history prior to the start of the execution as depicted in Fig. 3 and the estimated in-bid price using (16) are used to calculate the FP and is defined by the (10).

$$
P(\text { out }- \text { of }- \text { bid } \mid \text { price })=P(\text { out }- \text { of }- \text { bid }) \prod_{j=1}^{n} P\left(\text { price }_{j} \mid \text { out }- \text { of }- \text { bid }\right)
$$

And also, Naive assumes the conditional probabilities of the independent variables [2]. Henceforth, $P($ out - of - bid $\mid$ price $)$ can be decomposed into product of sums by using (11), where price $_{j}$ represents $n$ attributes as $\left\{\right.$ price $_{1}$, price $_{2}, \ldots$, price $\left._{n}\right\}$ that are conditionally independent of one another given (out - of - bid) as output.

Runtime Estimation A stochastic approach [6] is used to determine the runtime of a SWf task on a particular instance type. Assuming the tasks to be stochastic variables, its distributions and dependencies needs to be analyzed. The total execution time of tasks depends on the several factors as shown in (12).

$$
T_{\text {total }}=T_{R}+T_{D}+T_{O}+T_{Q}+T_{E}
$$

- $T_{R}$ is the resource initialization time

- $T_{D}$ is the data transfer time

- $\quad T_{O}$ is the resource overhead time based on the SWf structure

- $T_{Q}$ is the task queuing time

- $T_{E}$ is the execution time of the task

When the tasks are independent, it would be easier to find the mean and variance of $T_{\text {total }}$ as shown in Equations (13) and (14) respectively:

$$
\begin{gathered}
E\left[T_{\text {total }}\right]=E\left[T_{R}\right]+E\left[T_{D}\right]+E\left[T_{O}\right]+E\left[T_{Q}\right]+E\left[T_{E}\right] \\
\operatorname{Var}\left[T_{\text {total }}\right]=\operatorname{Var}\left[T_{R}\right]+\operatorname{Var}\left[T_{D}\right]+\operatorname{Var}\left[T_{O}\right]+\operatorname{Var}\left[T_{Q}\right]+\operatorname{Var}\left[T_{E}\right]
\end{gathered}
$$

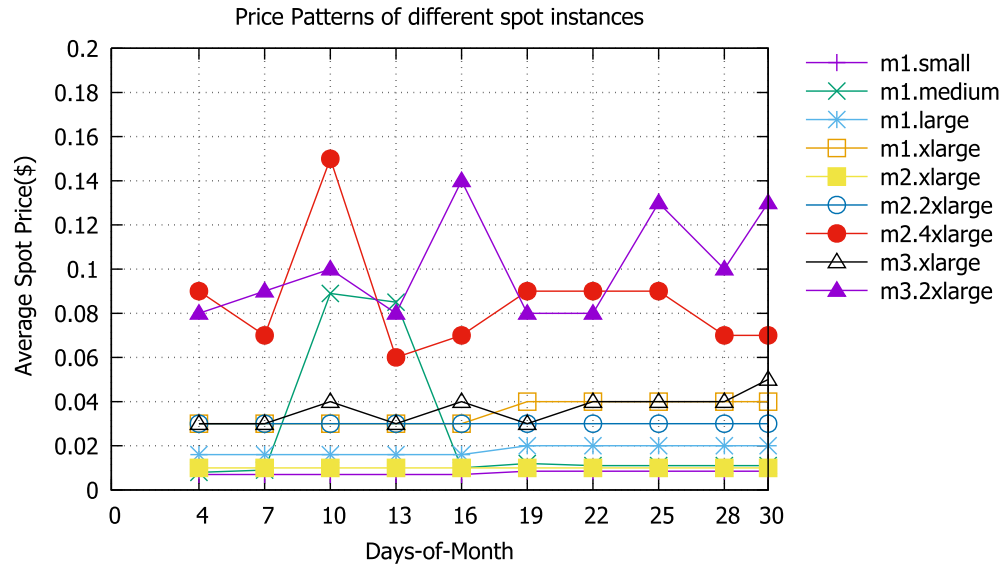

Fig. 3 Nov 2016 Month Patterns of Spot Price in US East (North Virginia) region 
However, if SWf tasks are dependent, considering the variance of $T_{\text {total }}$ is inappropriate. Hence, a covariance term is required to compute $\operatorname{Var}\left[T_{\text {total }}\right]$. Especially, this is related to the dependence between tasks execution time $T_{E}$ and data transfer time $T_{D}$

The stochastic model determines the total execution time of the tasks when fitting it to the observed data and is represented by (15).

$$
T_{\text {total }}=\mu(\chi, \beta)+\sigma(\chi, \beta) \xi(\chi, \beta)
$$

where $\chi$ is the task execution arguments, $\beta$ the unknown regression parameters, $\mu(\chi, \beta)$, $\sigma(\chi, \beta)$ and $\xi(\chi, \beta)$ are the stochastic variables. The regression problem consists in estimating unknown parameters $\beta$, thus estimating the mean, the variance, and the conditional distribution of the tasks runtime.

Bidding Strategy When there is no idle instances to schedule tasks, then the optimal inbid strategy is used to estimate bid prices of spot and blockspot instances and is defined by the following (16).

$$
\begin{gathered}
\text { in }- \text { bid }=e^{\alpha} * P_{B S}+\left(1-e^{\alpha}\left(\tau * P_{B S}+(1-\tau) * P_{S}\right)\right) \\
\text { where } \alpha=\frac{(-\sigma(L T B S-C T))}{F P}
\end{gathered}
$$

- $\quad P_{S}$ is the current spot price

- $\quad P_{B S}$ is the blockspot price

- $\quad L T B S$ is the Latest Time to Block Spot

- $\quad F P$ is the failure probability of the preceding in-bid price using Naive Bayes model

- $\quad C T$ is the Current Time

- $\sigma$ denotes how much higher the in-bid price as compared to spot price

- $\tau$ denotes how fast the in-bid price reaches close to the blockspot price

As the in-bid price increases and reach close to the blockspot price due to $C T$ reaching closer to $L T B S$ is attributed to $\tau$ parameter. When the value of $\tau$ increases, then the in-bid

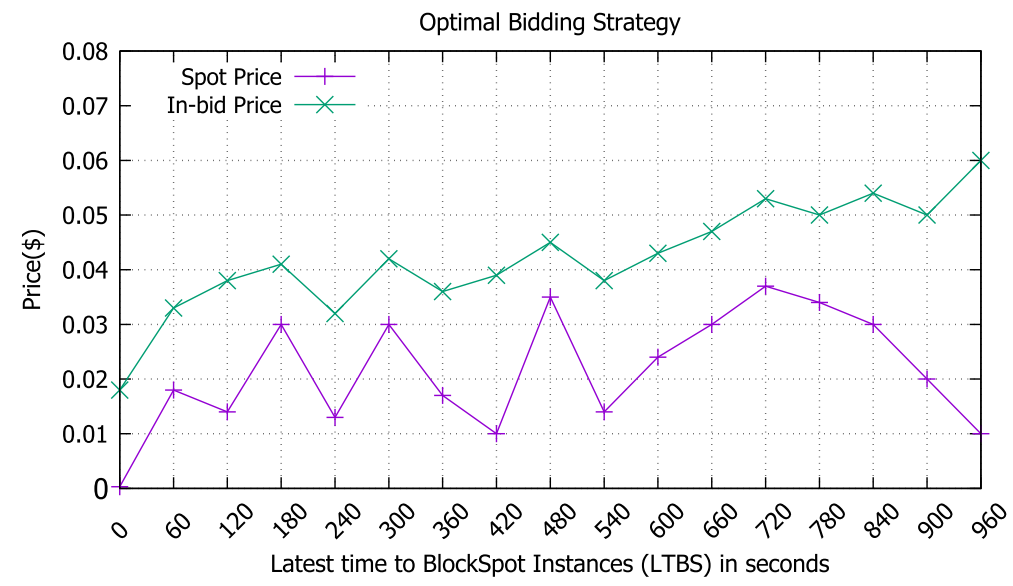

Fig. 4 Generation of In-bid Price value using Optimal Bidding Strategy 
price also increases and reaches closer to the blockspot price. The $F P$ of the previous inbid price is also considered as a feedback to the current in-bid price to optimize the bidding strategy. The in-bid price is calculated using the (17) and increases gradually with the SWf execution and as the $C T$ moves closer to the $L T B S$. Initially, the in-bid starts with a spot price and ends closer to the blockspot price. The reason behind in increasing the in-bid price is to minimize the risk of out-of-bid event as the SWf execution nears the LTBS and the deadline constraint is not violated. Figure 4 shows the in-bid price generated by the optimal bidding strategy.

\section{Proposed approaches}

The problem addressed in this work is to schedule SWf tasks onto the appropriate heterogeneous VM types with a combination of spot and blockspot hybrid instances in order to minimize the execution cost of SWf and fault-tolerant without violating the deadline.

Assumptions Data transfer cost between the VM types is assumed to be zero, since most of the public cloud services data transfer cost between the VMs is zero. It is also assumed that the scheduled tasks has complete access to VMs and hence there is no preemption.

Task replication technique is the most widely adopted fault-tolerant mechanism for enhancing the reliability and availability of services. In this paper, the proposed heuristic

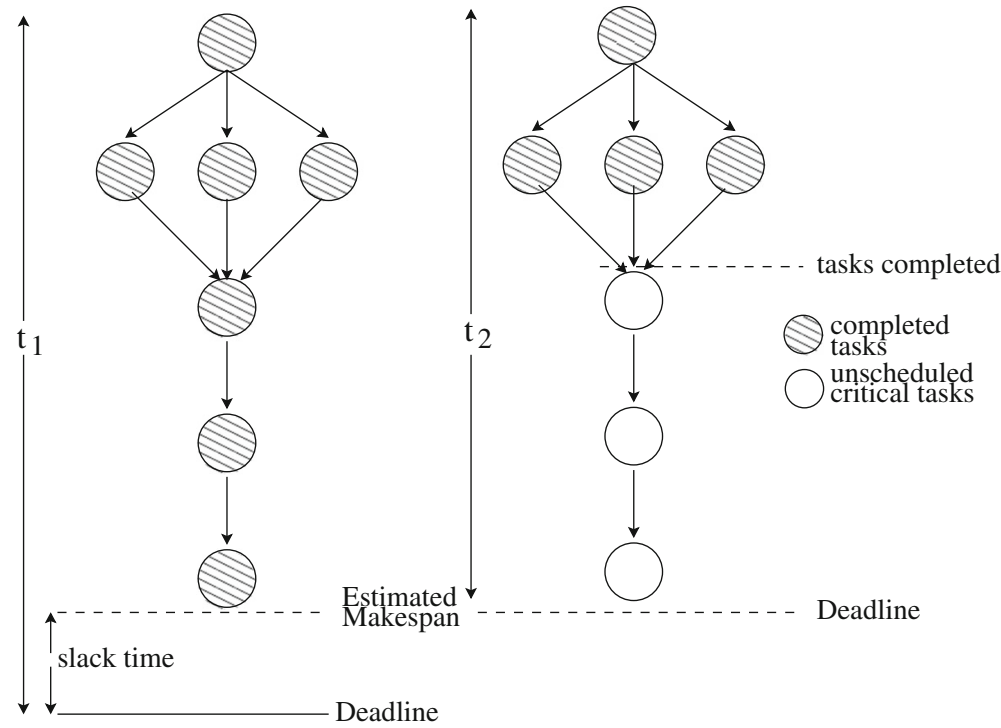

(a)

(b)

Fig. 5 Figure (a) shows a SWf at time $t_{1}$, where there is sufficient slacktime and in such circumstances the tasks are scheduled onto spot instances. Figure (b) depicts a SWf at time $t_{2}$, where there is no slacktime and some tasks are completed. Under these conditions, critical tasks are scheduled onto blockspot instances to ensure tasks execution without interruption 
uses task replication to mitigate the failures during SWf execution. As shown in Fig. 5b, in contemplation of meeting the SWf deadline, the unscheduled critical tasks are scheduled onto instances that offer low execution time and also replicated on spot instances.

Scheduling SWf tasks onto heterogeneous resources is an NP-hard problem [21]. Therefore, an efficient scheduling algorithm is proposed that schedules the ready tasks onto the best suitable resources in order to minimize the makespan and execution cost and also fault tolerant against resource failures. Once the scheduler receives the tasks, it estimates the critical path $C_{p}$ and the $C_{p}$ is usually different for every different instance types. After every execution of tasks, its $C_{p}$ weight is made zero and for the next successive ready tasks $C_{p}$ time is recomputed. Based on the SWf deadline and the estimated $C_{p}$ time, the LTBS time flag is computed. The difference between LTBS and the current time $C T$ leads to the type of resource required for the execution of tasks.

The proposed approaches are categorised into four possible scenarios. In scenarios 1 and 2, the tasks are mapped onto the spot instances since the LTBS is ahead of the $C T$ and also has enough slacktime to complete SWf execution before the deadline. Scenario 1 depicts the tasks mapping onto already running spot instances and in scenario 2 tasks are assigned to new spot instances due to the unavailability of running instances. In scenario 3 , and scenario 4, when the LTBS is before the $C T$, then the algorithm chooses blockspot instances to provide fault tolerance since there is no slacktime and also tasks are replicated either on spot or blockspot instances based on the CT, LTBS and deadline of the SWf. For convenience, the notations used in the algorithms is given in Table 2.

\subsection{Scenario 1: scheduling SWf task onto already running spot instances}

A scenario where the LTBS is ahead of the $C T$, then the proposed algorithm schedules the tasks onto spot instances first due to lower price as compared to blockspot and on-demand instances, and even if it fails due to out-of-bid events, there is still sufficient slacktime to rerun the tasks that have undergone failure. Before scheduling onto spot instances, the algorithm first finds the idleslots among the already running spot instances in order to maximize the resource utilization. Idle slots are the unused idle time periods of instances before the end of their charged period. Algorithm 1 details the methodology of finding these idle slots for scheduling.

Table 2 The Notations used in the Algorithm Design

\begin{tabular}{ll}
\hline Parameters & Definitions \\
\hline$R E$ & Runtime estimation \\
$S L$ & Schedule list \\
$L T B S$ & Latest time to block spot \\
$F P$ & Failure probability \\
$W_{D}$ & Workflow deadline \\
$C_{p}$ & Critical path \\
$T C T$ & Task completion time \\
$C T$ & Current time \\
\hline
\end{tabular}




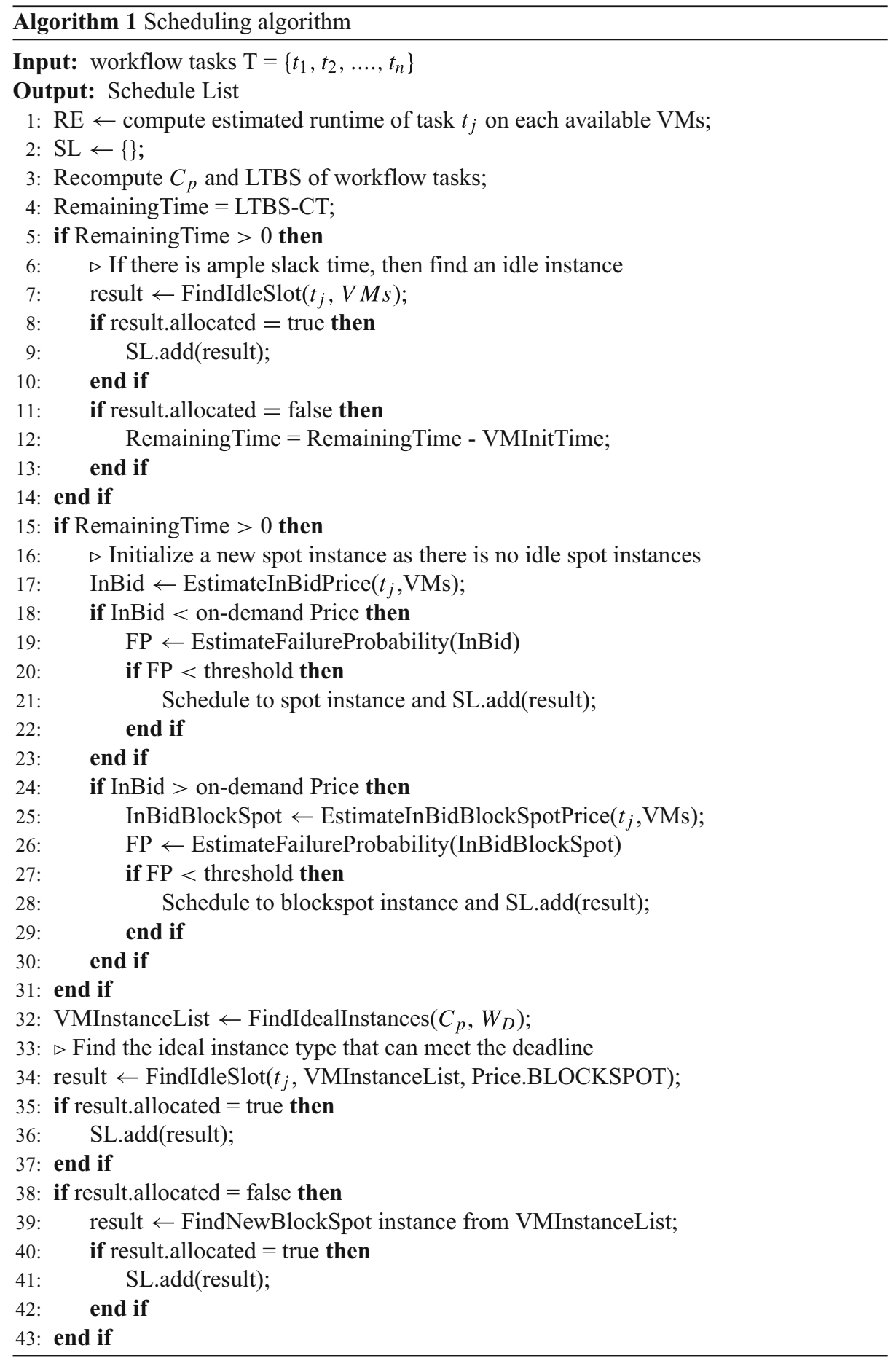




\subsection{Scenario 2: scheduling SWf task onto a new spot instances}

In the event when there are no such idle slots, then the optimal in-bid strategy is used to estimate the spot prices as discussed in the previous section and the FP of the previous in-bid price is also considered along with the in-bid price to optimize the bidding strategy. If the FP is less than the threshold, then that particular spot instance is considered for scheduling of task.

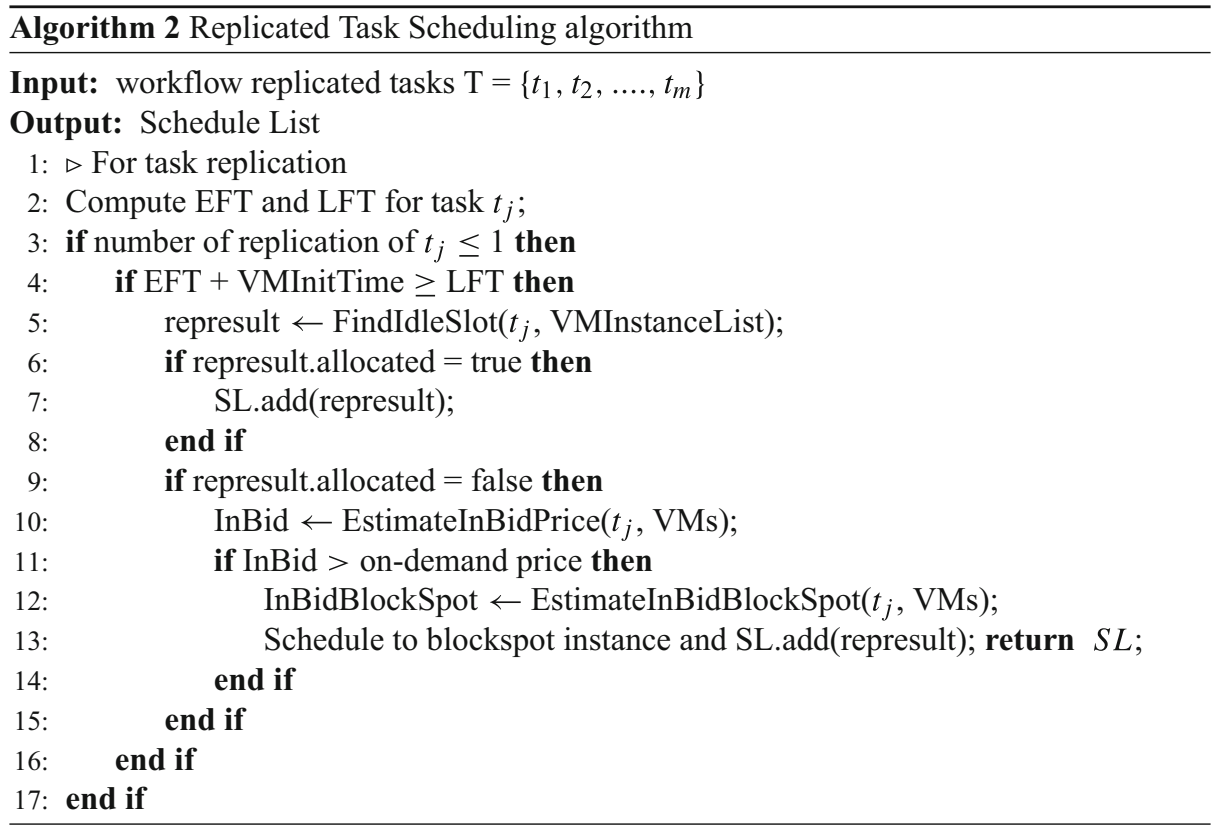

\subsection{Scenario 3: scheduling SWf task to an blockspot instances}

When LTBS is behind the $C T$ or the in-bid price reaches to the on-demand price or the FP is higher than the threshold, then instead of scheduling the tasks to an on-demand instance, a blockspot instance is used whose price is significantly lower than the on-demand instance. The optimal in-bid strategy is once again used when the spot price is higher than the ondemand to estimate the in-bid blockspot price and the FP of that particular blockspot is estimated. Once the blockspot is procured through bid, then the chances of being out-of-bid is negligible since it runs for either 1 hour to 6 hours without termination. Before finding an instance, a list of ideal instance types that can meet the SWf deadline are detailed in Algorithm 3.

\subsection{Scenario 4: scheduling replicated tasks under strict deadline}

Critical tasks of the SWf are replicated in order to provide fault tolerance. Algorithm 2 depicts its working. When the LTBS has crossed the CT, and also the tasks that do not have sufficient slack time during such situation all the critical tasks are replicated to meet the 
deadline. This scenario is similar to the previous one, first the idle slots are found between the instances that have not been used to schedule the replicated tasks. If no such idle slots exist, then it is scheduled on new spot or blockspot instances. Furthermore, during resource failure, the failed tasks are resubmitted as a ready tasks to the scheduler. Finally, using the optimal bidding strategy a in-bid price of spot instance is estimated and if it is less than the on-demand instance, then the spot instance is instantiated for replication of tasks. If not, then the optimal bidding strategy is used to estimate in-bid price of blockspot instance for scheduling of replicated tasks.

In the method FindIdealInstances, critical tasks are determined for each instance type. Usually, critical tasks vary for different instance types since tasks have different runtimes on different instances. Therefore, Algorithm 3 estimates the critical path $C_{p}$ for each instance types and maintains a list. This computation is performed initially and when the task completes its execution, then the executionengine verifies whether the task was critical and if it is, then the $C_{p}$ for those instances are recomputed. This increases the efficiency and avoids computing $C_{p}$ for each task. Once the $C_{p}$ of tasks are computed, then the Algorithm 3 adds the transfer time and task runtime for all the tasks on the $C_{p}$. Finally, the total $C_{p}$ time is computed, and if it is less than the remaining SWf deadline $W_{D}$, then the instance type is added to the selected instance list. This instance list is a list of instance types that obey with the deadline constraint.

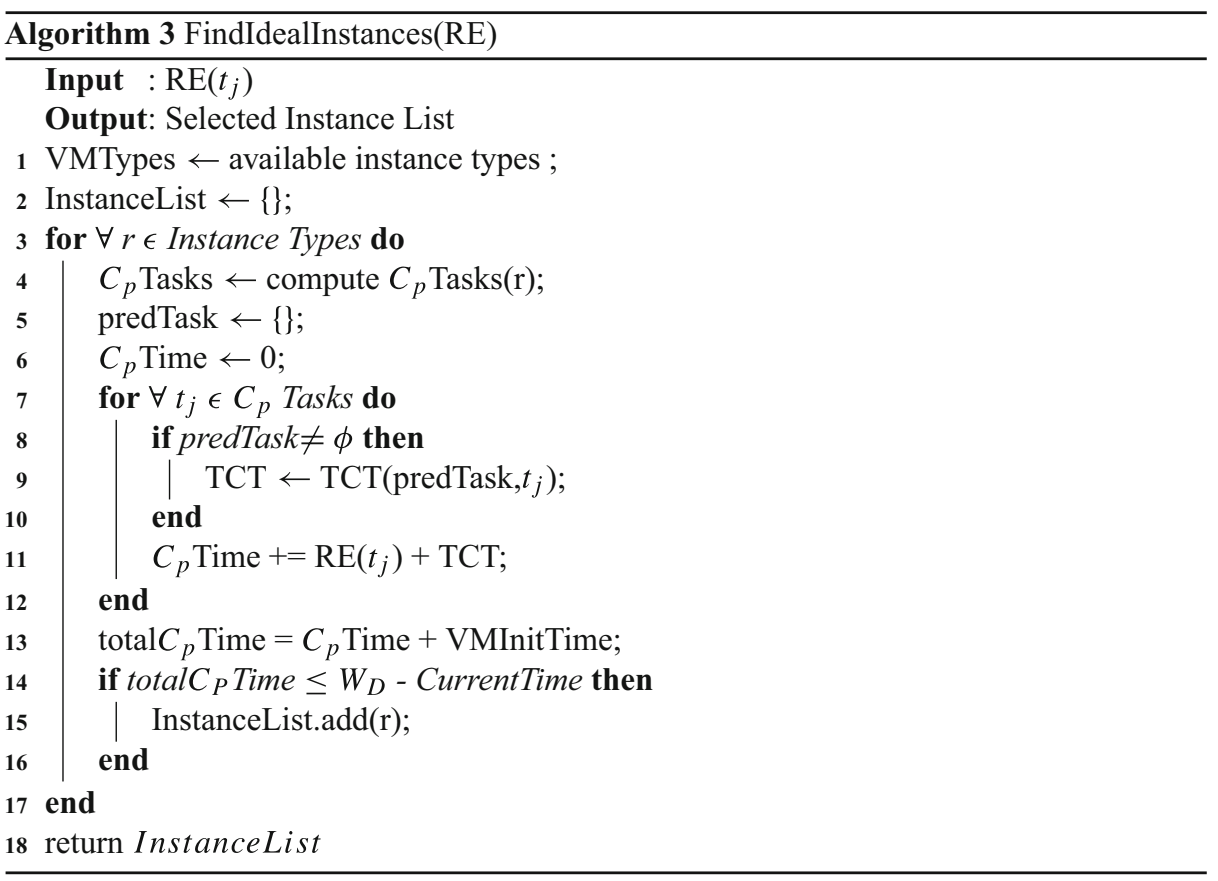

\section{Performance evaluation}

In this work CloudSim [3] is used to simulate the proposed approach in a cloud environment. It is extended to model the Amazon Web Services and it uses spot and blockspot market traces to simulate the spot prices. 
Application Modeling The Montage and LIGO SWf consisting of 1000 tasks are considered. The SWf patterns and its characteristics are explained in [14].

Resource Modeling The cloud computing resources are modelled similar to Amazons EC2 instances. Nine instance types ( $\mathrm{m} 1$.small, $\mathrm{m} 1$.medium, m1.large, $\mathrm{m} 1$.xlarge, m3.xlarge, $\mathrm{m} 3$.2xlarge, m2.xlarge, $\mathrm{m} 2$.2xlarge, $\mathrm{m} 2.4 x$ large) for spot, blockspot and on-demand are considered. And the pricing of these instances is adopted from Linux based instances of the Amazons US East (Northern Virginia) region. The spot price history is also taken from this same region for a period from $1^{\text {st }}$ to $30^{\text {th }}$ November 2016 . The average spot and blockspot price and its frequency of being out-of-bid per month, i.e., (Low/High)) and its cost savings over on-demand are tabulated in Table 1.

Failure Modeling In this paper, failures are modeled using Weibull distribution [18]. We have assumed that once the resources are failed during the execution are not used again. And furthermore, the failures are considered to be independent and the distribution models the time to failure of a particular resource. The parameters of the Weibull distribution are modeled similar to the parameters used in [14].

Scheduling Algorithms In order to compare the proposed heuristics and optimal bidding strategy, a full on-demand scheduling (ODS) and spot instance scheduling (SIS) is developed that works similar to the proposed approach, but in ODS all the ready tasks are scheduled onto on-demand instances only and in SIS with naive bidding strategy only spot instances are used for scheduling of tasks. The proposed heuristics uses Spot and Block Spot Instances (SBSIS) schduling with an optimal bidding strategy for scheduling to achieve high fault-tolerance and cost-effectiveness.

\subsection{Results and analysis}

In this section, we analyze the performance of our proposed approach by evaluating them on the parameters of fault tolerance, makespan, cost, and resource utilization. We have run 1000 tasks each of Montage and LIGO SWf with different deadlines. The deadlines have been classified as strict (10000-30000), moderate (40000-60000) and relaxed (70000-90000) deadlines in seconds for Montage and similarly strict (1000 - 4000), moderate (5000 $10000)$ and relaxed $(11000-15000)$ deadlines in seconds for LIGO SWf respectively. In next subsection, we present the performance and analysis of our proposed heuristics with regard to different parameters.

\subsubsection{Fault tolerance}

The main objective of the proposed algorithm is to provide fault-tolerant scheduling. Figure 6a and $b$ shows the failure rate of Montage and LIGO SWf with respect to different deadlines. It can be observed that under strict deadlines, the proposed algorithm has shown significantly low failure rate as compared to without fault-tolerant approach. However, during relaxed deadline both the algorithms have a substantial low failure rate. It is evident from the results that the proposed heuristics has higher fault-tolerance and due to fact that both performs similar under relaxed deadlines.

\subsubsection{Evaluation of makespan}

Makespan is the turnaround time of the SWf execution. We attempt to reduce it, especially under the strict deadline. Figure $7 \mathrm{a}$ and $\mathrm{b}$ shows the performance of our algorithms with 


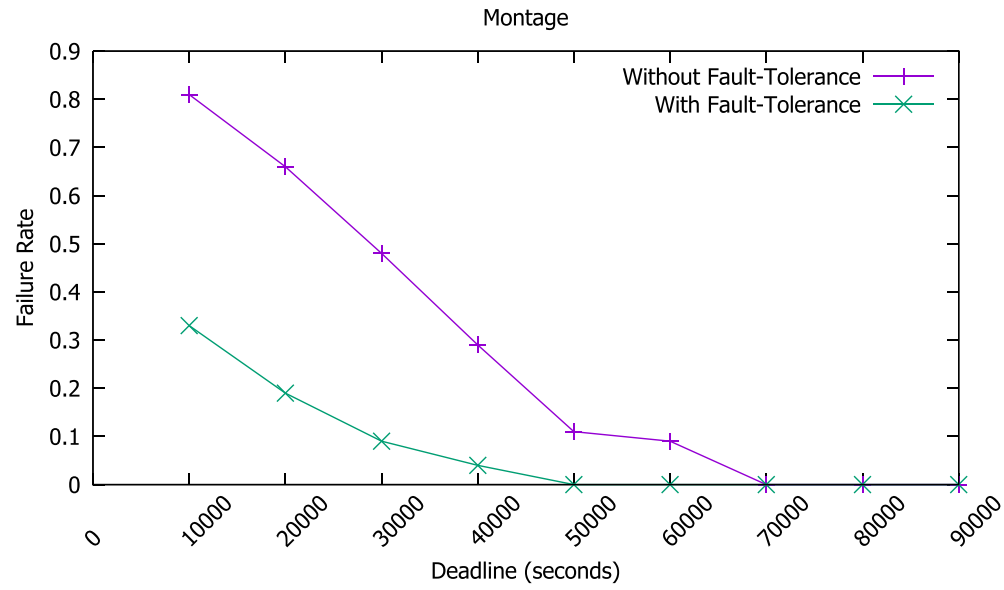

(a) Failure rate of Montage at different Deadline Intervals

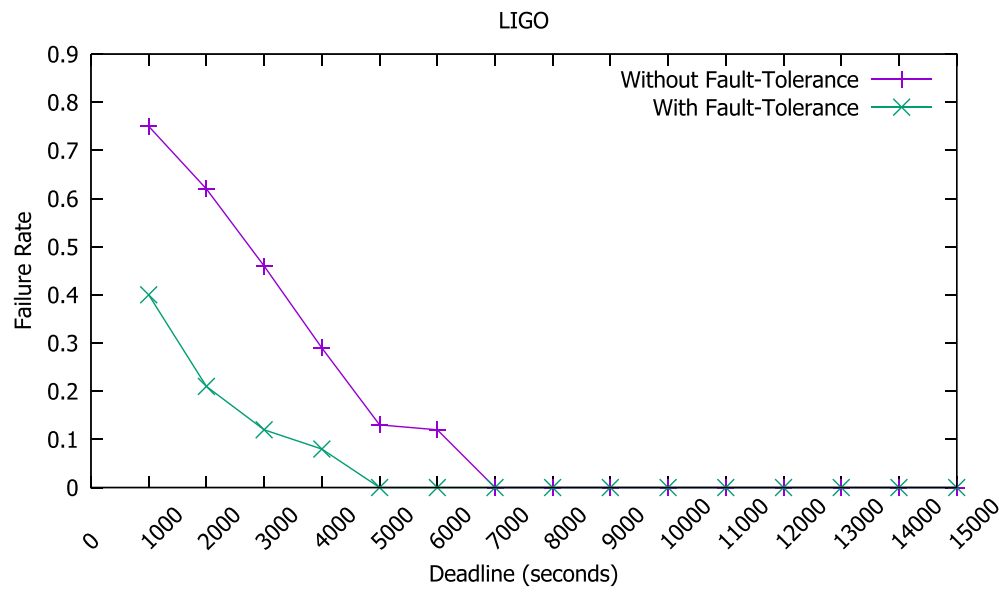

(b) Failure rate of LIGO at different Deadline Intervals

Fig. 6 Failure Rate of Algorithms with different Deadline Intervals

regard to makespan. The proposed heuristic i.e., Spot and Blockspot Instance Scheduling (SBSIS) as discussed in Section 6 in comparison with the On-Demand Scheduling (ODS) and Spot Instance Scheduling (SIS) outperforms SIS and relatively similar to ODS. In SIS, spot instances frequently face out-of-bids and due to which overheads and restarts are high resulting an increase in makespan. But in the case of ODS, resource availability and reliability is high and hence there is reduction in makespan and also meets the strict deadlines, the SBSIS uses both spot and blockspot instances to induce fault-tolerance and minimization in makespan.

\subsubsection{Effect on cost}

The proposed algorithms use spot and blockspot instances in comparison with on-demand instance to reduce the execution cost. It is evident from the Fig. 8a and b that the proposed 


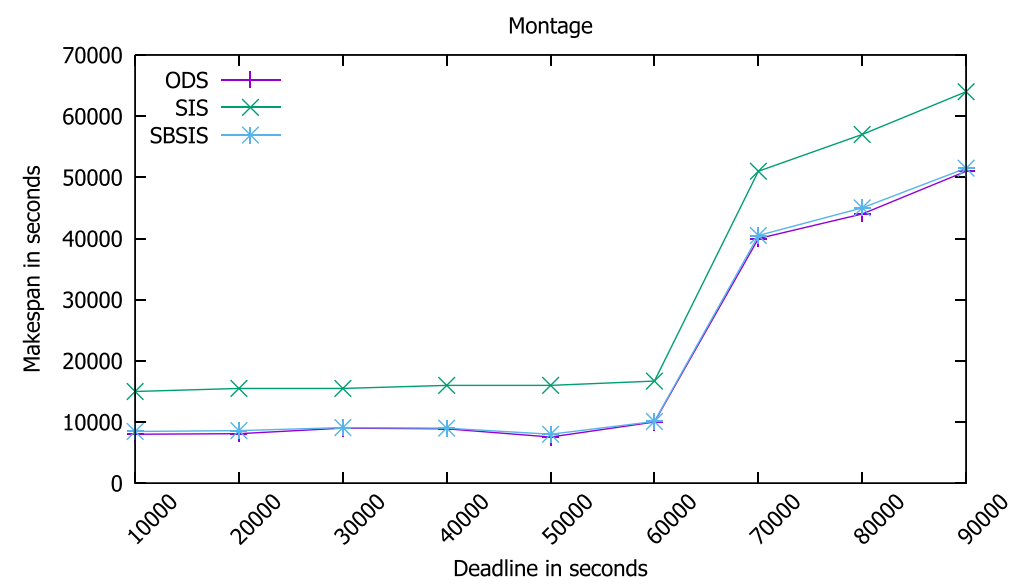

(a) Makespan of Montage at different Deadline Intervals

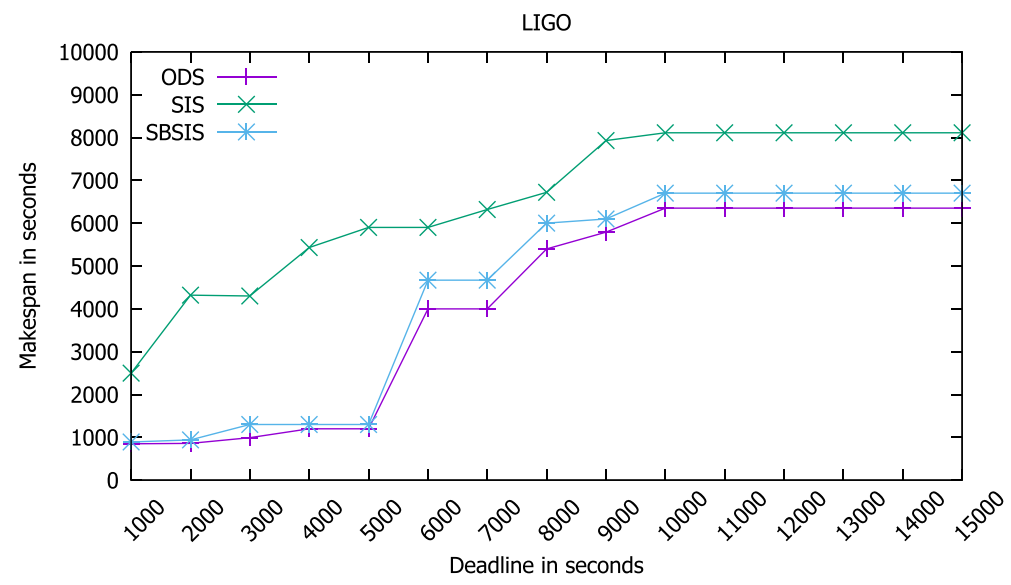

(b) Makespan of LIGO at different Deadline Intervals

Fig. 7 Makespan of SWf with different Deadline Intervals

algorithm has reduced the execution cost significantly when compared with SIS and ODS. Due to frequent out-of-bids in SIS and higher resource cost in ODS has led to higher execution cost. But in the case of proposed SBSIS, both spot and blockspot instances are used efficiently and its resource cost is cheaper as compared with on-demand instances. Further, during the out-of-bid of spot instances rather than scheduling the tasks to on-demand instances, tasks are scheduled onto blockspot since these instances run for at least 1hour or 6 hours without termination with lower cost.

\subsubsection{Effectiveness of checkpointing}

Checkpointing is one of the techniques used in fault-tolerant mechanisms. Figure $9 \mathrm{a}$ and $\mathrm{b}$ demonstrates the effectiveness of checkpointing. In the proposed approach, four different checkpoints are used with variations in spot prices. The variations of spot price are done by 


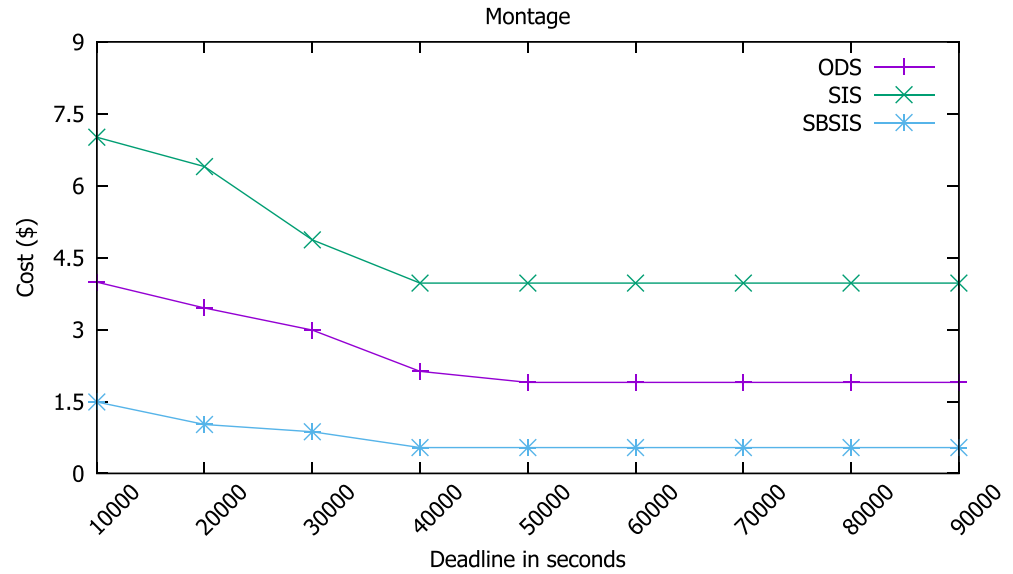

(a) Cost of Montage at different Deadline Intervals

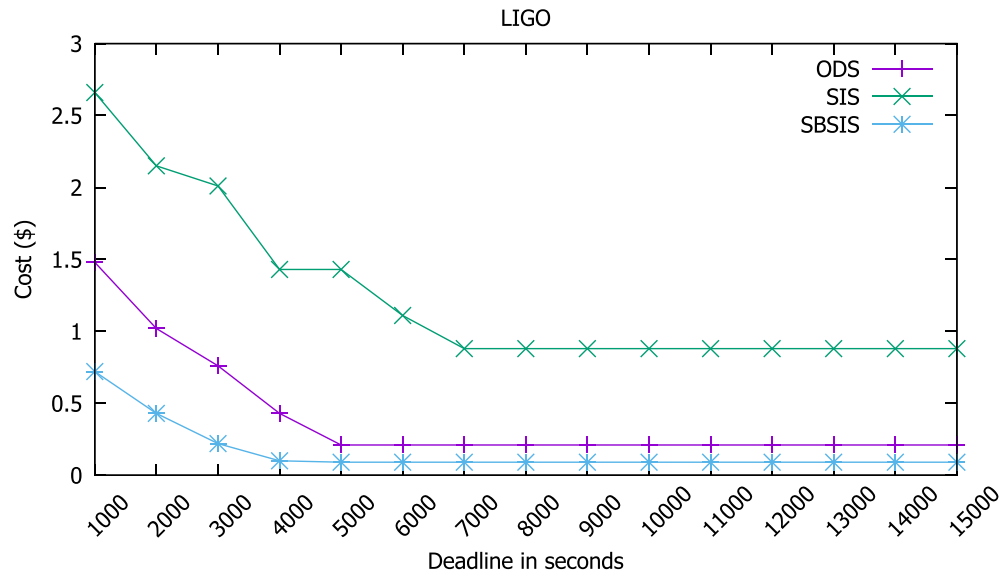

(b) Cost of LIGO at different Deadline Intervals

Fig. 8 Cost of SWf with different Deadline Intervals

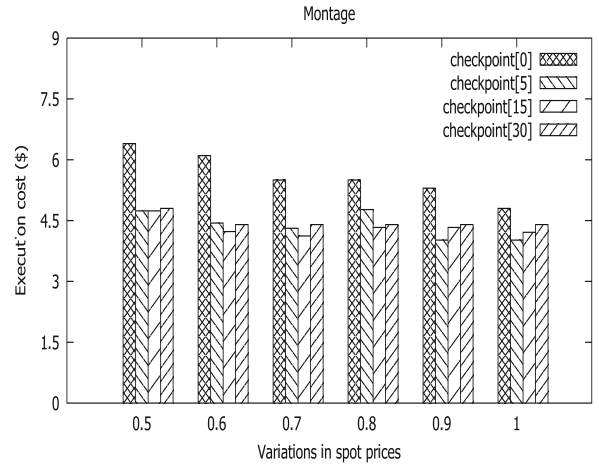

(a) Montage

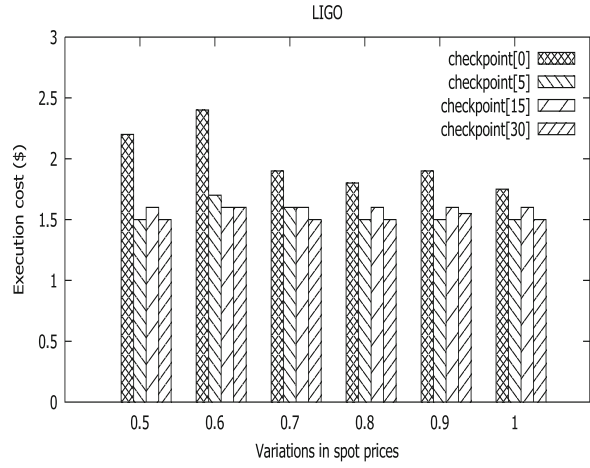

(b) LIGO

Fig. 9 Execution Cost with different checkpointing 
changing the scale of inter price time, i.e., the time between two spot prices. Checkpoint [0] indicates no checkpointing where 5 minutes checkpoint [5], checkpoint [15] and checkpoint [30] indicates checkpointing after 5,15 and 30 minutes respectively. It is observed that when there is no checkpointing, the execution cost is high and with checkpoint [5], checkpoint [15] and checkpoint [30] the execution cost is comparatively low but there is not much significant difference among checkpoints.

\subsubsection{Effectiveness of task replication}

Figure 10a and $\mathrm{b}$ shows the number of tasks replicated by each algorithm with different deadline intervals. It is evident that as the deadline tends to relax, then the number of replications of tasks is also reduced and it is more significant for proposed heuristic SBSIS and SIS. The tasks are not replicated in ODS due to high availability of on-demand instances

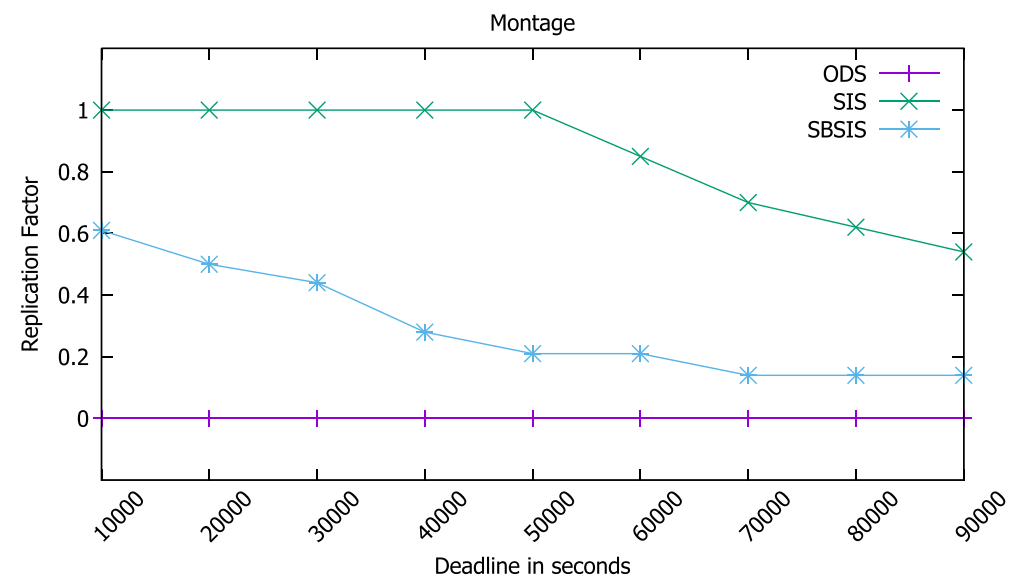

(a) Replication Factor of Montage at different Deadline Intervals

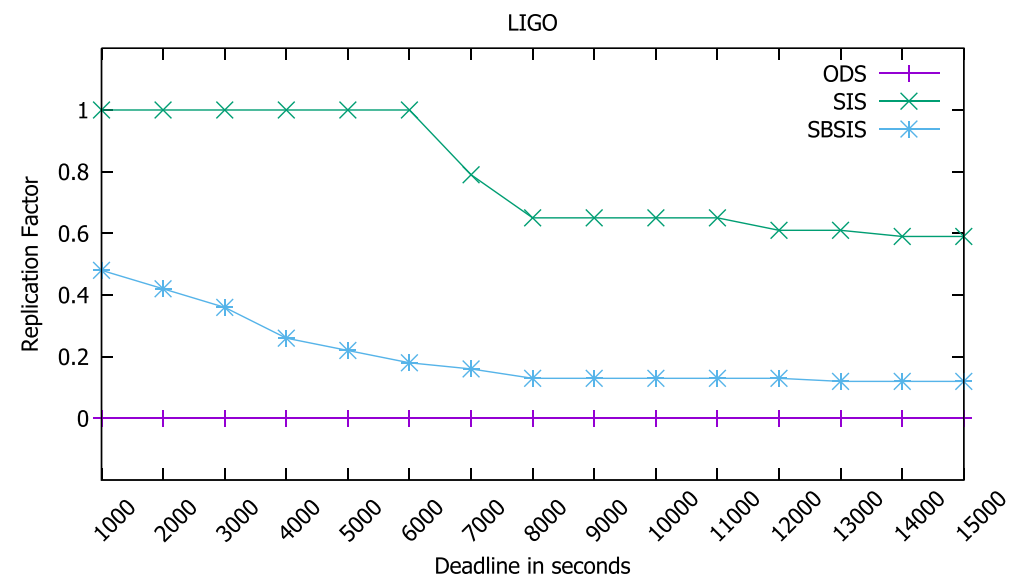

(b) Replication Factor of LIGO at different Deadline Intervals

Fig. 10 Replication Factor of SWf with different Deadline Intervals 
and do not fail during execution. But in SIS and SBSIS, tasks are replicated depending on the spot instances out-of-bid and CT of tasks with respect to deadlines. However, in SBSIS heuristic blockspot instances are used along with spot instances to mitigate replication of tasks.

\section{Conclusions and future directions}

In this paper, a scheduling heuristic that schedules SWf tasks onto spot and blockspot in comparison with on-demand instances are presented. The main objective of this paper is to minimize execution cost of SWf and fault-tolerant during out-of-bid events. An optimal bidding strategy that bids in correspondence to SWf execution to reduce cost is also presented. Simulation experiments are conducted to evaluate the performance of the proposed heuristics. The heuristics fare well in estimating the runtime of the SWf tasks, finding ideal instances for scheduling, estimating in-bid price of spot and blockspot instances, failure probability of in-bid price using Amazon's spot price history, an optimal bidding strategy to acquire instances that costs less and having minimal out-of-bid events, replication of critical tasks during strict deadline, different checkpoints to reduce tasks failure rate and execution cost.

Future research direction includes enhancing the proposed heuristics considering data transfer cost between instances and adoption of machine learning algorithms to further increase the proposed heuristics robustness with respect to makespan, cost and fault tolerance.

\section{References}

1. Almi'Ani K, Lee YC (2016) Partitioning-based workflow scheduling in clouds. In: 2016 IEEE 30th international conference on Advanced information networking and applications (AINA). IEEE, Piscataway, pp 645-652

2. Bala A, Chana I (2015) Intelligent failure prediction models for scientific workflows. Expert Syst Appl 42(3):980-989

3. Calheiros RN, Ranjan R, Beloglazov A, De Rose CAF, Buyya R (2011) Cloudsim: a toolkit for modeling and simulation of cloud computing environments and evaluation of resource provisioning algorithms. Software: Practice and experience 41(1):23-50

4. Calheiros RN, Buyya Rajkumar (2014) Meeting deadlines of scientific workflows in public clouds with tasks replication. IEEE Trans Parallel Distrib Syst 25(7):1787-1796

5. Chen J, Yang Y (2007) Adaptive selection of necessary and sufficient checkpoints for dynamic verification of temporal constraints in grid workflow systems. ACM Transactions on Autonomous and Adaptive Systems (TAAS) 2(2):6

6. Chirkin AM, Kovalchuk SV (2014) Towards better workflow execution time estimation. IERI Procedia 10:216-223

7. Darbha S, Agrawal DP (1994) A task duplication based optimal scheduling algorithm for variable execution time tasks. In: International conference on parallel processing, 1994. ICPP 1994, vol 2. IEEE, Piscataway, pp 52-56

8. Dejun J, Pierre G, Chi C-h (2010) Ec2 performance analysis for resource provisioning of service-oriented applications. In: Service-Oriented computing. ICSOC/ServiceWave 2009 workshops. Springer, Berlin, pp 197-207

9. Díaz JL, Entrialgo J, García M, García J, García DF (2017) Optimal allocation of virtual machines in multi-cloud environments with reserved and on-demand pricing. Futur Gener Comput Syst 71:129-144

10. Hwang S, Kesselman C (2003) Grid workflow: A flexible failure handling framework for the grid. In: 2003. Proceedings. 12th IEEE International Symposium on High Performance Distributed Computing. IEEE, Piscataway, pp 126-137

11. Jangjaimon I, Tzeng N-F (2015) Effective cost reduction for elastic clouds under spot instance pricing through adaptive checkpointing. IEEE Trans Comput 64(2):396-409 
12. Javadi B, Abawajy J, Buyya R (2012) Failure-aware resource provisioning for hybrid cloud infrastructure. J Parallel Distrib Comput 72(10):1318-1331

13. Lifka D, Foster I, Mehringer S, Parashar M, Redfern P, Stewart C, Tuecke S (2013) Xsede cloud survey report. Technical report, National Science Foundation, USA, Tech. Rep.

14. Juve G, Chervenak A, Deelman E, Bharathi S, Mehta G, Vahi K (2013) Characterizing and profiling scientific workflows. Futur Gener Comput Syst 29(3):682-692

15. Li J, Humphrey M, Cheah Y-W, Ryu Y, Agarwal D, Jackson K, van Ingen C (2010) Fault tolerance and scaling in e-science cloud applications: Observations from the continuing development of modisazure. In: 2010 IEEE Sixth International Conference on e-Science (e-Science). IEEE, Piscataway, pp 246-253

16. Li X, Zhang L, Wu Y, Liu X, Zhu E, Yi H, Wang F, Zhang C, Yang Y (2017) A novel workflow-level data placement strategy for data-sharing scientific cloud workflows. IEEE Trans Serv Comput

17. Mehmi S, Verma HK, Sangal AL (2016) Comparative analysis of cloudlet completion time in time and space shared allocation policies during attack on smart grid cloud. Procedia Computer Science 94:435440

18. Plankensteiner K, Prodan R, Fahringer T, Kertész A, Kacsuk P (2009) Fault detection, prevention and recovery in current grid workflow systems. In: Grid and services evolution, pp 1-13

19. Qu C, Calheiros RN, Buyya R (2016) A reliable and cost-efficient auto-scaling system for web applications using heterogeneous spot instances. J Netw Comput Appl 65:167-180

20. Ribas M, Furtado CG, de Souza JN, Barroso GC, Moura A, Lima AS, Sousa FRC (2015) A petri netbased decision-making framework for assessing cloud services adoption The use of spot instances for cost reduction. J Netw Comput Appl 57:102-118

21. Rodriguez MA, Buyya R (2014) Deadline based resource provisioningand scheduling algorithm for scientific workflows on clouds. IEEE Transactions on Cloud Computing 2(2):222-235

22. Samak T, Gunter D, Goode M, Deelman E, Juve G, Silva F, Vahi K (2012) Failure analysis of distributed scientific workflows executing in the cloud. In: Proceedings of the 8th international conference on network and service management, pp 46-54 international federation for information processing

23. Tang X, Li K, Liao G (2014) An effective reliability-driven technique of allocating tasks on heterogeneous cluster systems. Clust Comput 17(4):1413-1425

24. Vinay K, Dilip Kumar SM (2016) Auto-scaling for deadline constrained scientific workflows in cloud environment. In: India Conference (INDICON) 2016 IEEE Annual. IEEE, Piscataway, pp 1-6

25. Wan J, Zhang R, Gui X, Xu B (2016) Reactive pricing: an adaptive pricing policy for cloud providers to maximize profit. IEEE Trans Netw Serv Manag 13(4):941-953

26. Zhu X, Ji W, Guo H, Zhu D, Yang LT, Liu L (2016) Fault-tolerant scheduling for real-time scientific workflows with elastic resource provisioning in virtualized clouds. IEEE Trans Parallel Distrib Syst 27(12):3501-3517

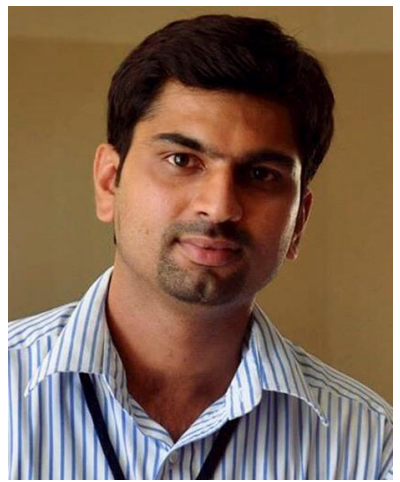

Vinay K. is a research scholar in the department of Computer Science and Engineering, University Visvesvaraya College of Engineering, Bangalore University, Bangalore, India. He received his Bachelor degree in Electronics and Communication from JNN College of Engineering, Visvesvaraya Technological University (VTU), Shimoga, India and Master degree from AMC College of Engineering, VTU, Bangalore, India. He has secured 1st Rank in his Masters Degree. His research interests include Cloud Computing, Big Data Analytics and Virtualization. He is a student member of IEEE. 


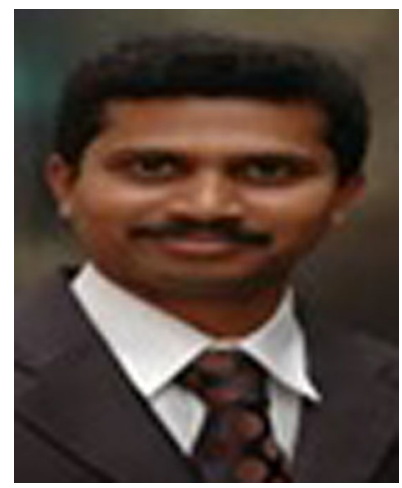

Dr. S. M. Dilip Kumar is an Associate Professor in Computer Science and Engineering at University Visvesvaraya College of Engineering, Bangalore. His research interests include Wireless Sensor Networks, Internet of Things and Cloud Computing. He has published nearly 50 papers in International Journals and Conferences and he was the recipient of the best paper award at ICHPCA 2014. He has completed a research project sponsored by Department of Science and Technology, Govt. of India in the area of grid computing and a consultancy project sponsored by Government of Karnataka in the area of Mobile Governance.

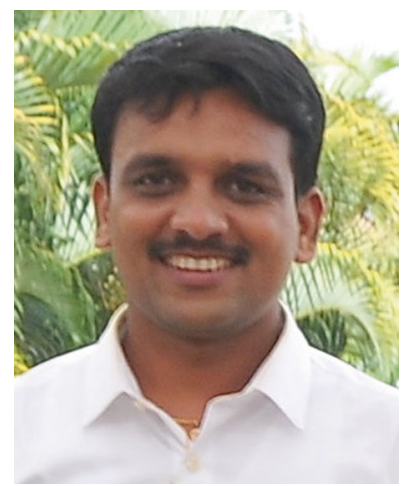

Raghavendra S. is a research scholar in the department of Computer Science and Engineering, University Visvesvaraya College of Engineering, Bangalore University, Bangalore, India. He received his Bachelor degree in Computer Science and Engineering from BMS Institute of Technology, Visvesvaraya Technological University (VTU), Bangalore, India and Master degree from R.V.College of Engineering, VTU, Bangalore, India. His research interests include Cloud Computing, applied cryptography and network security. He is a student member of the IEEE. 


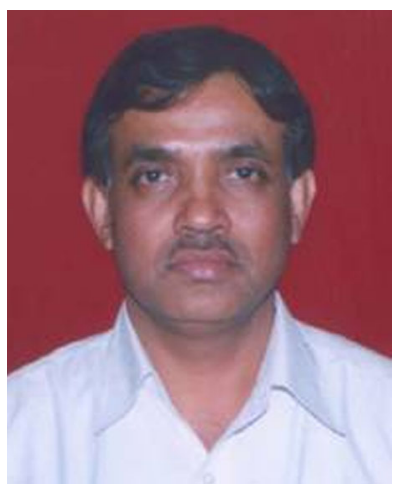

Dr. Venugopal K. R. is currently the Principal, University Visvesvaraya College of Engineering, Bangalore University, Bangalore. He obtained his Bachelor of Engineering from University Visvesvaraya College of Engineering. He received his Masters degree in Computer Science and Automation from Indian Institute of Science Bangalore. He was awarded Ph.D. in Economics from Bangalore University and Ph.D. in Computer Science from Indian Institute of Technology, Madras. He has a distinguished academic career and has degrees in Electronics, Economics, Law, Business Finance, Public Relations, Communications, Industrial Relations, Computer Science and Journalism. He has authored and edited 65 books on Computer Science and Economics, which include Petrodollar and the World Economy, C Aptitude, Mastering C, Microprocessor Programming, Mastering $\mathrm{C}++$ and Digital Circuits and Systems etc.. He has filed 101 Patents. During his three decades of service at UVCE he has over Author Biographies Click here to download Author Biographies biography.docx 500+ research papers to his credit. His research interests include Computer Networks, Wireless Sensor Networks, Parallel and Distributed Systems, Digital Signal Processing and Data Mining. 